\title{
Transcutaneous Vagus Nerve Stimulation is Associated with Lower Mechanical Ventilation and Mortality in COVID-19 Patients
}

\author{
Patrick Nemechek ${ }^{1 *}$, Guido Antonelli², and Ariel Braida ${ }^{2}$ \\ ${ }^{1}$ Nemechek Autonomic Medicine, Buckeye Arizona, USA \\ ${ }^{2}$ Hospital Zonal Virgen del Carmen de Zárate, Zárate, Buenos Aires, Argentina
}

*Corresponding author: Patrick Nemechek, Nemechek Autonomic Medicine, Buckeye Arizona, USA, E-mail: dr@autonomicmed.com

Received: 24 May, 2021 | Accepted: 19 Jul, 2021 | Published: 29 Jul, 2021

Citation: Nemechek P, Antonelli G, Braida A (2021) Transcutaneous Vagus Nerve Stimulation is Associated with Lower Mechanical Ventilation and Mortality in COVID-19 Patients. J Emerg Dis Virol 6(2): dx.doi.org/10.16966/2473-1846.165

Copyright: (c) 2021 Nemechek P, et al. This is an open-access article distributed under the terms of the Creative Commons Attribution License, which permits unrestricted use, distribution, and reproduction in any medium, provided the original author and source are credited.

\section{Abstract}

Objective: Evaluate the safety and efficacy of transcutaneous vagus nerve stimulation in preventing respiratory failure and improving survival in hospitalized COVID-19 patients.

Design, Setting, and Participants: Final analysis of an ongoing single-arm, uncontrolled open-label, and observational trial to assess the transcutaneous vagus nerve stimulation (tVNS) in hospitalized SARS-CoV-2 infected subjects. Eligible subjects are 18 years old or older, requiring hospitalization for COVID-19 pneumonia.

Results: The 51 subjects enrolled with a mean age of 53.6 (22-85) years. Mechanical ventilation was required in only $3(5.9 \%)$ cases. Of those receiving mechanical ventilation, 1 survived, and 2 died. Of all 51 hospitalized patients, $9(17.6 \%)$ subjects died, with 7 cases (13.7\%) directly attributable to respiratory failure. Relative to other reported COVID-19 hospitalization outcomes data, our mortality rate of $17.6 \%$ (9 of 51) is lower than many other reported cohorts $10.2 \%-50 \%$. Only $5.9 \%$ (3 of 51) of subject's required mechanical ventilation compared to $7.4 \%-79 \%$ of subjects requiring mechanical ventilation in other cohorts. Adverse events associated with tVNS occurred in only 2 subjects (3.4\%) and consisted of reversible oral paresthesia and orthostatic hypotension.

Conclusion and Relevance: Our study evaluated the impact of tVNS in hospitalized patients diagnosed with COVID-19 pneumonia. The use of tVNS resulted in a low rate of adverse events, infrequent mechanical ventilation, and a high rate of survival. Several other studies using various therapies have ranges of mortality (14-50\%) and the requirement of mechanical ventilation (7-79\%). Considering the study was done in a resource-limited public hospital, it is important to note that the mortality rate and the frequency of mechanical ventilation were less than other reported COVID-19 cohorts. Only mild and self-limiting adverse events tVNS occurred in 2 subjects highlighting the safety of vagus stimulation.

Keywords: Vagus nerve stimulation; VNS; SARS-CoV-2; COVID-19; Mechanical ventilation; Mortality; Cytokine storm; Cytokine release syndrome

Abbreviations: VNS: Vagus Nerve Stimulation; CRS: Cytokine Release Syndrome; IL: Interleukin; TNF: Tumor Necrosis Factor; CAP: Cholinergic Anti-inflammatory Pathway; ARDS: Acute Respiratory Distress Syndrome

\section{Introduction}

As the severe acute respiratory syndrome coronavirus 2 (SARSCoV-2) pandemic continues and thousands of individuals die daily from COVID-19 worldwide, a more effective therapeutic option is essential. Infection with SARS-CoV-2 is characterized by a dramatic cytokine storm in some patients with COVID-19 [1]. Often referred to as Cytokine Release Syndrome (CRS), this storm is due to the release of high levels of pro-inflammatory cytokines such as interleukin (IL)-1 $\beta$, IL-6, tumor necrosis factor (TNF), chemokines by respiratory epithelial, dendritic cells, and macrophages. The surge of pro-inflammatory cytokines has the potential to contribute to the multitude of pathological changes seen in severe COVID-19 patients (Figure 1).
We hypothesize that CRS can be dampened with a subsequent improvement in the patient's clinical outcome by activating the vagaldriven cholinergic anti-inflammatory pathway (CAP). Boezaart AP, et al., published a case report of two individuals with COVID-19 who both experienced significant reductions in IL-6 and CRP levels using 60 minutes of taVNS per day. Both individuals had COVID-19 pneumonia and were essentially free of respiratory symptoms by the end of the treatment course (16 and 18 days) with taVNS [2]. Additionally, Staats P, et al. reported on the clinical improvement in two individuals with SARS-CoV-2 respiratory symptoms [3].

First described by Borovikova and Tracey, the CAP controls cytokine release via afferent vagal signaling into the dorsal vagal complex (DVC) [4,5]. The DVC is responsible for receiving and 


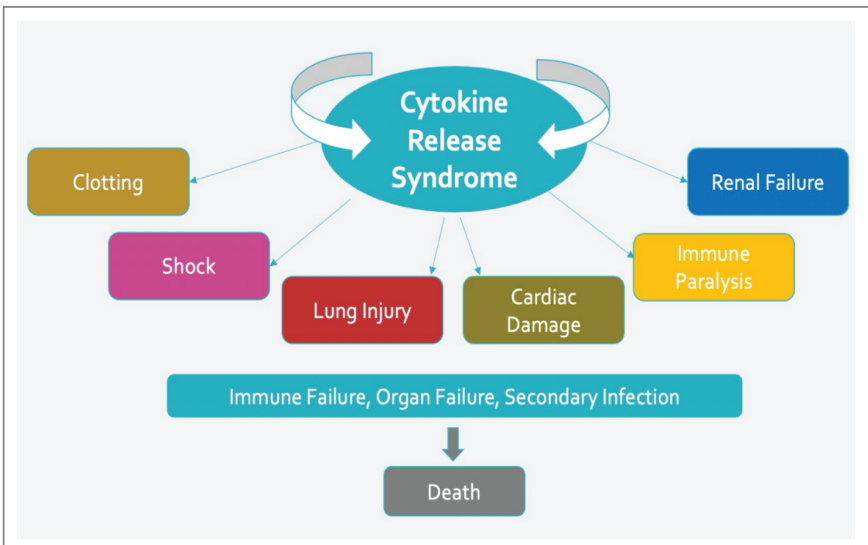

Figure 1: Potential negative impact of cytokine release syndrome.

processing that vagal afferent signaling [4-6]. After the impulse is analyzed by the DVC, efferent impulses via sympathetic (spinal cord) and parasympatheitic (vagus nerve) pathways result in the release of acetylcholine $(\mathrm{ACh})$ secretion at the site of injury that terminates the release of pro-inflammatory cytokines.

Individuals may have suppressed vagus nerve functionality partially reflected in low heart rate variability (HRV). Depressed vagal tone leads to functional impairment of CAP resulting in excessive cytokine distribution (cytokine storm) followed by tissue injury, pulmonary dysfunction, ARDS, and immune suppression, whereas in the case of ANS balance, controlled cytokine distribution is generating tissue repair and virus elimination.

The CAP is a neural reflex capable of decreasing the release of proinflammatory cytokines and is modulated by the vagus nerve. Electric neuromodulation of the vagus nerve inhibits TNF synthesis during endotoxemia, ischemia/reperfusion injury, hemorrhagic shock, septic peritonitis, and other excessive cytokine disorder modulation during endotoxemia ischemia/reperfusion injury, hemorrhagic shock, septic peritonitis, and other excessive cytokine disorders [7].

Vagus nerve stimulation (VNS) can play an essential role in managing patients with SARS-CoV-2 infection [7]. The vagus nerve widely innervates multiple organs, especially the lungs and gastrointestinal tract. When the electrical stimulation is applied transcutaneously at the ear (taVNS), afferent fibers are stimulated, thereby activating the central autonomic network and resulting in activation of the efferent fibers of both the right and left vagus nerves as well as sympathetic fibers within the spinal cord and potential activation of the HPA axis [8]. The cumulative effect of ta VNS is the improvement of the inflammatory modulation [7]. taVNS therapy is uncomplicated, safe, inexpensive, and treatment might suppress the CRS enough to improve the clinical course in COVID-19 patients requiring hospitalization (Figure 2).

An observational trial was designed to explore the safety and potential benefit of transcutaneous auricular vagus nerve stimulation in hospitalized patients infected with COVID-19 [9-12].

\section{Methods}

\section{Study design and participants}

This is an open-label, observational trial designed to see if taVNS stimulation was safe and might improve clinical outcomes for patients hospitalized with COVID-19 pneumonia. The protocol was approved by the ethical committee of Hospital Zonal Virgen del Carmen de

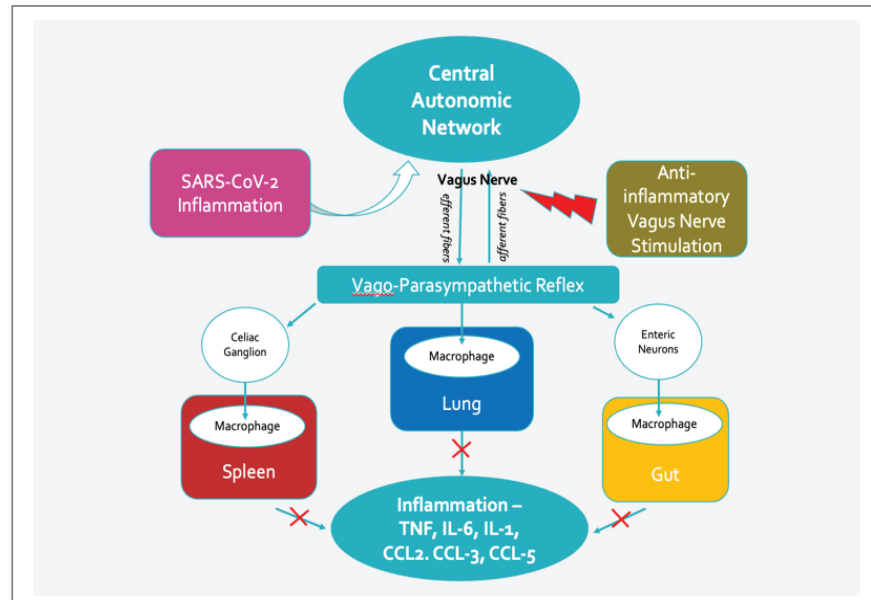

Figure 2: Potential beneficial effect of vagus nerve stimulation in COVID-19 patients.

Zárate, Zárate, Buenos Aires, Argentina. Written informed consent was obtained from all individuals before enrollment.

Eligible subjects are 18 years old or older, have a positive or presumed positive test for SARS-CoV-2, and require hospitalization for symptoms consistent with COVID-19 pneumonia. Study enrollment began in July 2020 with a planned minimal enrollment of 50 subjects. A total of 51 subjects were enrolled.

\section{Inclusion criteria}

1. Patients older than 18 years of age.

2. Tested positive or suspected/presumed positive for SARS-CoV-2 infection.

3. Patients requiring hospital admission with evidence of pneumonia and/or pneumopathy.

4. Patients showing fever or respiratory symptoms with radiological findings of pneumonia or pneumopathy.

5. Respiratory distress ( $\geqq 30$ breaths/ $\mathrm{min}$ ) or Oxygen saturation $\leq$ $93 \%$ at rest in ambient air; or oxygen saturation $\leq 97 \%$ with $\mathrm{O}_{2}$ $>5 \mathrm{~L} / \mathrm{min}$.

6. The patient provides signed and witnessed Informed Consent.

\section{Exclusion criteria}

1. Already enrolled in an experimental trial for SARS-CoV-2 or COVID-19 therapy.

2. Potentially life-threatening heart rhythm.

3. Pregnancy or potential pregnancy.

4. Current implantation of an electrical and/or neurostimulator device, including but not limited to a cardiac pacemaker or defibrillator, vagal neurostimulator, deep brain stimulator, spinal stimulator, bone growth stimulator, or cochlear implant.

5. History of an aneurysm, intracranial hemorrhage, brain tumors, or significant head trauma.

6. Belongs to a vulnerable population or has any condition such that his or her ability to provide informed consent, comply with the follow-up requirements, or provide self-assessments is compromised (e.g., homeless, developmentally disabled, and prisoner). 


\section{Intervention with vagus nerve stimulation}

After obtaining written consent, patients were assigned to receive 5 minutes of transcutaneous vagus nerve stimulation (tVNS) with an electronic neuromodulation device (Vitality Smart cable, Nemechek Technologies, figure 3). Stimulation parameters are $10 \mathrm{~Hz}, 800 \mathrm{uS}$, biphasic pulse wave with the voltage set at $10 \mathrm{v}$ below the perception threshold) and was applied every 6 hours for up to 14 days.

The electrical stimulation was delivered through a small clip that makes contact within the concha and the back of the ear (Figure 4). The ear and the clip contact points are cleaned with an alcohol swab before each treatment. After the placement of the clip, the voltage was slowly increased until the subject could perceive the current. The voltage was set just below the level of perception by slowly reducing the current until it was no longer detectable by the subject.

All other therapies (antivirals, steroids, immunoglobulin) as determined by the managing physician were allowed.

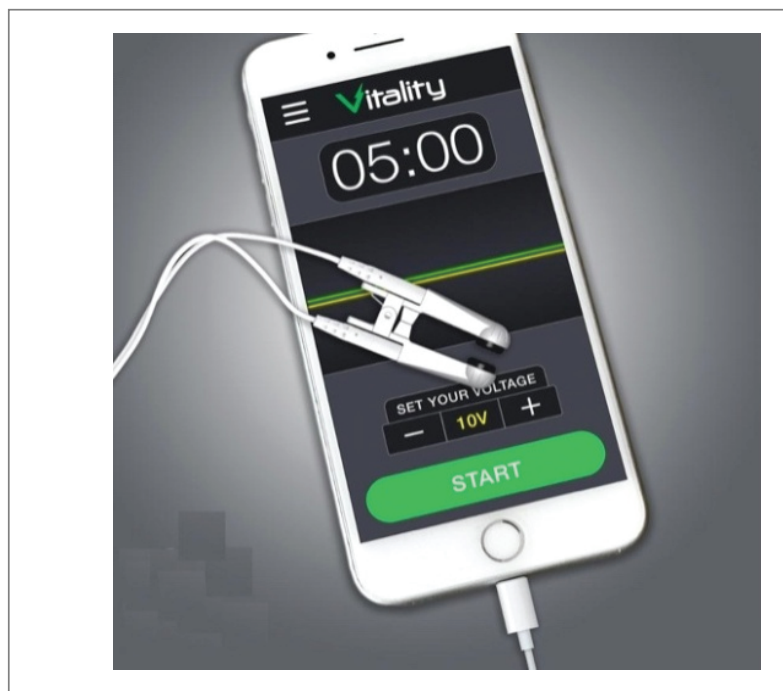

Figure 3: taVNS is powered and controlled by iPod (Apple, Inc.)

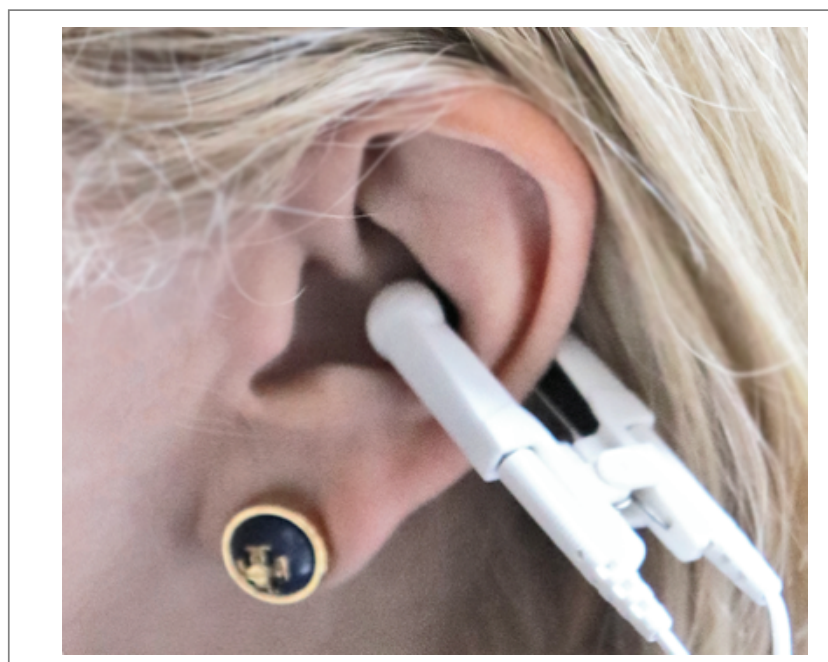

Figure 4: Concha Clip Placement.

\section{Outcomes measures}

Primary outcome measures are as follows:

1. Survival without the need for mechanical ventilation on day 14 . [time frame: 14 days from symptom onset] is to be considered a positive outcome.

a. Patients needing mechanical ventilator utilization (including non-invasive Ventilation, NVI) or death will be considered a negative outcome.

b. New do not resuscitate (DNR) orders will be considered a negative event at the date of the DNR.

2. Survival [time frame: 14 days].

a. Overall survival.

3. Cumulative incidence of successful tracheal extubation (defined as duration extubation $>48 \mathrm{~h}$ ) at day 14 [time frame: 14 days].

a. Death or DNR order will be considered a competing negative event.

\section{Results}

\section{Study participants}

Between July $7^{\text {th }}$ and September $1^{\text {st }}, 2020,51$ subjects ( 29 men and 22 women) with a mean age of 53.6 years (22-85) were enrolled in the study. The majority of subjects had comorbidities that included hypertension (66.7\%), diabetes (18\%), cardiovascular disease (11.8\%), obesity (25.5\%), and chronic kidney disease (5.9\%). The mean oxygen saturation level on admission was 93.2\% [range 79-98\%], and oxygen saturation levels reached a low mean of $90.2 \%$ [range 79-97] during the hospitalization (Table 1).

Thirty-four subjects (66.6\%) were treated with tVNS 4 times per day for 5 minutes. Because of a staffing emergency during an outbreak of COVID-19 amongst hospital staff, an additional 17 subjects (33.3\%) only received tVNS only twice daily during their hospital stay. Survival between 2 versus 4 tVNS treatments per day was nearly identical (Table 2).

In addition to $\mathrm{VVNS}$, all subjects received oxygen and dexamethasone, and 9 subjects (17.6\%) received hyperimmune globulin as the only other forms of an investigational therapy for COVID-19 and were equally split between those receiving tVNS BID versus QID.

\section{Survival}

Of the initial 51 subjects treated with tVNS, 82.4\% $(n=42)$ survived, with only $3.4 \%(n=1)$ of the survivors requiring mechanical ventilation. Of the 9 non-survivors, 7 died from respiratory failure attributed to COVID-19 pneumonia, and only 2 received mechanical ventilation. Two non-survivors died from diabetic ketoacidosis, and bacterial septicemia felt to be related to advance AIDS.

Of the 51 survivors, $97.6 \%(n=41)$ did so without the need for mechanical ventilation. There was no significant difference in the oxygen saturation levels at the time of admission between survivors on non-survivors. Survivors had more comorbid diagnoses than non-survivors, but the numbers are too small to draw any statistical conclusions.

Both survivors and non-survivors had comorbid diagnoses, but a higher number of comorbid conditions were seen in those not requiring mechanical ventilation. 
Table 1: Comparison of survivors and non-survivors.

\begin{tabular}{|l|c|c|}
\hline & Survivor (\%) & Non-Survivor (\%) \\
\hline Of Total Patients & $42(82.4)$ & $9(17.6)$ \\
\hline Rec'dVNS & $42(100)$ & $9(100)$ \\
\hline Admission O sat\%, Mean & $79-98 \%[$ mean 93.2] & $87-97 \%$ [mean 92.0] \\
\hline No Mech. Vent. & $41(97.6)$ & $7(77.8)$ \\
\hline Rec'd Mech. Vent. & $1(3.4)$ & $2(22.2)$ \\
\hline Co-Morbidity & & \\
\hline Obesity & $11(26.2)$ & $2(22.2)$ \\
\hline Diabetes Mellitus & $13(31.0)$ & $5(55.6)$ \\
\hline Hypertension & $27(64.3)$ & $7(77.8)$ \\
\hline CAD/CVA & $4(9.5)$ & $1(11.1)$ \\
\hline Renal & $2(4.8)$ & $1(11.1)$ \\
\hline Asthma/COPD & $3(7.1)$ & $1(11.1)$ \\
\hline HIV & - & $1(11.1)$ \\
\hline
\end{tabular}

Table 2: Comparison of 2 versus 4 tVNS treatments per day.

\begin{tabular}{|l|c|c|}
\hline & VNS BID (\%) & VNS QID (\%) \\
\hline Of Total Patients & $17(36.2)$ & $30(63.8)$ \\
\hline Admission $\mathrm{O}_{2}$ sat\%, Mean & $85-97 \%$ [mean 93.8] & $79-98 \%$ [mean 92.3] \\
\hline Survive & $14(82.4)$ & $24(80.0)$ \\
\hline Mortality & $3(17.6)$ & $6(20.0)$ \\
\hline No mech. Vent. & $41(97.6)$ & $7(77.8)$ \\
\hline Rec'd Mech. Vent. & $1(3.4)$ & $2(22.2)$ \\
\hline
\end{tabular}

\section{Mechanical ventilation}

Patients treated with tVNS had a low need for mechanical ventilation. Of all 51 subjects, only 3 (5.9\%) received mechanical ventilation. Of subjects not receiving mechanical ventilation, overall survival was $85.4 \%$ (Table 3 ).

\section{Adverse events}

Adverse events associated with tVNS occurred in only 2 subjects (3.9\%) and consisted of oral paresthesia and orthostatic hypotension. Both of which were described as mild by the managing physician and resolved spontaneously. These did not occur with the use of a lower voltage during subsequent treatments with vagal stimulation. Both patients with AE from tVNS survived. It is unknown if these adverse effects were directly attributable to taVNS

\section{Comparison to other cohorts}

A control group is ideally used to provide a relative standard against which therapy is compared. Because of the outbreak of SARS-CoV-2 among hospital staff and the rapidly escalating number of SARS-CoV-2 cases arriving within the hospital, the study was modified to limited staff contact with infected patients unless clinically necessary, and the control group was eliminated from the study. As such, a comparison to other reported cohort data on overall mortality and the frequency of mechanical ventilation is outlined. The data is stratified according to the number of hospitalized subjects reported for each cohort.

Although our analysis details only treated patients, one can see that overall mortality is either lower or similar to other reported cohorts when vagus nerve stimulation is incorporated in the treatment regimen (Table 4) [13-20].

We are aware that comparison with each different cohort should be made with caution as we are unable to delineate the relative available treatments between each of these reported cohorts. That being said, the patients in each of these cohorts received a wide variety of treatments reflecting the resources available and the prevailing treatment approaches taken by each cohort group.

\section{Discussion and Conclusion}

While vaccination against SARS-CoV-2 improves hospitalization and mortality rates in wealthier countries, shortages of SARS-CoV-2 vaccines and basic medical supplies such as oxygen are still leading to widespread death from COVID-19 [21]. The use of a vagus nerve stimulator offers a treatment option that holds the potential to alter the course of the infection in hospitalized individuals infected with SARS-CoV-2.

Implementation of tVNS as a treatment option can be instituted quickly within any hospital. Technicians can be trained very quickly,

Table 3: Comparison of patients requiring or not requiring mechanical ventilation.

\begin{tabular}{|l|c|c|c|}
\hline & $\begin{array}{c}\text { Mechanical } \\
\text { Ventilation (\%) }\end{array}$ & $\begin{array}{c}\text { Non-Mechanical } \\
\text { Ventilation (\%) }\end{array}$ & Total (\%) \\
\hline All patients & $3(5.9)$ & $48(94.1)$ & 51 \\
\hline Survival-Day 14 & $1(33.3)$ & $41(85.4)$ & $42(82.4)$ \\
\hline Mortality & $2(66.7)$ & $7(14.6)$ & $9(17.6)$ \\
\hline Admission O2 sat\% & $\begin{array}{c}93-98 \%,[\text { mean } \\
95.3 \%]\end{array}$ & $79-99 \%[$ mean 93.0] & \\
\hline Co-Morbidity & $1(33.3)$ & $12(25)$ & $13(25.5)$ \\
\hline Obesity & $1(33.3)$ & $17(35.4)$ & $18(35.3)$ \\
\hline Diabetes Mellitus & $3(100)$ & $31(64.6)$ & $34(66.7)$ \\
\hline Hypertension & $1(33.3)$ & $5(10.4)$ & $6(11.8)$ \\
\hline CAD/CVA & - & $3(6.3)$ & $3(5.9)$ \\
\hline Renal & $1(33.3)$ & $3(6.3)$ & $4(7.8)$ \\
\hline Asthma/COPD & & & \\
\hline
\end{tabular}

Table 4: Comparison of mortality and mechanical ventilation rates among other published cohorts.

\begin{tabular}{|l|c|c|c|}
\hline \multicolumn{1}{|c|}{ Cohort } & $\begin{array}{c}\text { Number of } \\
\text { Hospitalized } \\
\text { patients }\end{array}$ & $\begin{array}{c}\text { Overall } \\
\text { Mortality (\%) }\end{array}$ & $\begin{array}{c}\text { Require } \\
\text { Mechanical } \\
\text { ventilation }\end{array}$ \\
\hline Seattle, U.S [8] & 24 & $12(50 \%)$ & $18(75 \%)$ \\
\hline Argentena - VNS & 51 & $9(17.6 \%)$ & $3(5.9 \%)$ \\
\hline China [9] & 191 & $54(28.2 \%)$ & $32(16.6 \%)$ \\
\hline New York, U.S [7] & 257 & $101(39 \%)$ & $203(79 \%)$ \\
\hline New York, U.S [11] & 393 & $40(10.2 \%)$ & $130(33 \%)$ \\
\hline Louisiana, U.S [10] & 1382 & $325(23.5 \%)$ & $364(26.3 \%)$ \\
\hline New York, U.S [12] & 1999 & $292(14.6 \%)$ & $445(22.2 \%)$ \\
\hline United Kingdom [13] & 6678 & $2212(33.1 \%)$ & $497(7.4 \%)$ \\
\hline United States [14] & 11721 & $2514(21.5 \%)$ & $1967(16.8 \%)$ \\
\hline
\end{tabular}


tVNS is safe, the devices are inexpensive, and can be run off an inexpensive smart phone. Similar to a fingertip pulse oximeter, a single device can be easily decontaminated and used among multiple patients.

Electrical stimulation of the vagus nerve can modulate the working point of the inflammatory response in a wide variety of potentially lethal medical conditions (endotoxemia, ischemia/reperfusion injury, hemorrhagic shock, septic peritonitis) [7]. A growing number of publications agree with our position that tVNS can blunt the surge of inflammatory cytokines found in COVID-19 pneumonia and could favorably alter the course of the illness.

Scientific purists will emphatically state that no treatment should be put into practice without fully controlled clinical trials. In general, I wholeheartedly agree with this principle, but the dire reality faced by many communities not yet vaccinated against SARS-CoV-2needs actionable therapeutic options immediately. Treatment of patients with tVNS offers them a potentially beneficial, safe, and inexpensive therapy when faced with little to no other options.

Our results suggest that transcutaneous vagus nerve stimulation (tVNS) in hospitalized patients requiring oxygen support or having respiratory distress is a safe, rapid, and technically simple process that could easily be deployed in developing countries. Because of the lack of a control group, it is difficult to comment on the efficacy accurately. We believe a well-designed controlled clinical trial of taVNS is warranted.

\section{References}

1. Ye Q, Wang B, Mao J (2020) The pathogenesis and treatment of the 'Cytokine Storm' in COVID-19. J Infect 80: 607-613.

2. Boezaart AP, Botha DA (2021) Treatment of Stage 3 COVID-19 With Transcutaneous Auricular Vagus Nerve Stimulation Drastically Reduces Interleukin-6 Blood Levels: A Report on Two Cases. Neuromodulation 24: 166-167.

3. Staats P, Giannakopoulos G, Blake J, Liebler E, Levy RM (2020) The Use of Non-invasive Vagus Nerve Stimulation to Treat Respiratory Symptoms Associated with COVID-19: A Theoretical Hypothesis and Early Clinical Experience. Neuromodulation 23: 784-788.

4. Borovikova LV, Ivanova S, Zhang M, Yang H, Botchkina GI, et al. (2000) Vagus nerve stimulation attenuates the systemic inflammatory response to endotoxin. Nature 405: 458-462.

5. Tracey K (2002) The inflammatory reflex. Nature 420: 853-859.

6. Tracey KJ (2007) Physiology and immunology of the cholinergic antiinflammatory pathway. J Clin Invest 117: 289-296.

7. Kaniusas E, Kampusch S, Tittgemeyer M, Panetsos F, Gines RF, et al. (2019) Current Directions in the Auricular Vagus Nerve Stimulation I-A Physiological Perspective. Front Neurosci 13: 854.

8. Liu CH, Yang MH, Zhang GZ, Wang XX, Li B, etal. (2020) Neural networks and the anti-inflammatory effect of transcutaneous auricular vagus nerve stimulation in depression. J Neuroinflammation 17: 54.
9. Wang D, Hu B, Hu C, Zhu F, Liu X, et al. (2020) Clinical Characteristics of 138 Hospitalized Patients with 2019 Novel Coronavirus-Infected Pneumonia in Wuhan, China. JAMA 323: 1061-1069.

10. Chen G, Wu D, Guo W, Cao Y, Huang D, et al. (2020) Clinical and immunologic features in severe and moderate Coronavirus Disease 2019. J Clin Invest 130: 2620-2629.

11. Zheng HY, Zhang M, Yang CX, Zhang N, Wang XC, et al. (2020) Elevated exhaustion levels, and reduced functional diversity of $T$ cells in peripheral blood may predict severe progression in COVID-19 patients. Cell Mol Immunol 17: 541-543.

12. Sun F, Kou H, Wang S, Yun L, Houyu Z, et al. (2020) Medication patterns and disease progression among 165 patients with coronavirus disease 2019 (COVID-19) in Wuhan, China: a singlecentered, retrospective, observational study. Preprints with the Lancet.

13. Cummings MJ, Baldwin MR, Abrams D, Jacobson SD, Meyer BJ, et al. (2020) Epidemiology, clinical course, and outcomes of critically ill adults with COVID-19 in New York City: a prospective cohort study. Lancet 395: 1763-1770.

14. Bhatraju PK, Ghassemieh BJ, Nichols M, Kim R, Jerome KR, et al. (2020) Covid-19 in Critically III Patients in the Seattle Region - Case Series. N Engl J Med 382: 2012-2022.

15. Zhou F, Yu T, Du R, Fan G, Liu Y, et al. (2020) Clinical course and risk factors for mortality of adult in patients with COVID-19 in Wuhan, China: a retrospective cohort study. Lancet 395: 1054-1062.

16. Price-Haywood EG, Burton J, Fort D, Seoane L (2020) Hospitalization and Mortality among Black Patients and White Patients with Covid-19. N Engl J Med 382: 2534-2543.

17. Goyal P, Choi JJ, Pinheiro LC, Schenck EJ, Chen R, et al. (2020) Clinical Characteristics of Covid-19 in New York City. N Engl J Med. 382: 2372-2374.

18. Petrilli CM, Jones SA, Yang J, Rajagopalan H, O'Donnell L, et al. (2020) Factors associated with hospital admission and critical illness among 5279 people with coronavirus disease 2019 in New York City: prospective cohort study. BMJ 369: $\mathrm{m} 1966$.

19. Docherty $A B$, Harrison EM, Green CA, Hardwick HE, Pius R, et al. (2020) Features of 20133 UK patients in hospital with covid-19 using the ISARIC WHO Clinical Characterisation Protocol: prospective observational cohort study. BMJ 369: m1985.

20. Fried MW, Crawford JM, Mospan AR, Watkins SE, Munoz B, et al. (2020) Patient Characteristics and Outcomes of 11,721 Patients with COVID19 Hospitalized Across the United States. Clin Infect Dis 72: e558-e565.

21. Pérez-Peña R (2021) Some hospitals don't have enough oxygen to keep patients alive. Here's why. The New York Times, USA. 